\title{
Good is not Good Enough: The Benchmark Stroke Door-to-Needle Time Should be 30 Minutes
}

\author{
Noreen Kamal, Oscar Benavente, Karl Boyle, Brian Buck, Ken Butcher, Leanne K. Casaubon, \\ Robert Côté, Andrew M Demchuk, Yan Deschaintre, Dar Dowlatshahi, Gordon J Gubitz, Gary Hunter, \\ Tom Jeerakathil, Albert Jin, Eddy Lang, Sylvain Lanthier, Patrice Lindsay, Nancy Newcommon, \\ Jennifer Mandzia, Colleen M. Norris, Wes Oczkowski, Céline Odier, Stephen Phillips, \\ Alexandre Y Poppe, Gustavo Saposnik, Daniel Selchen, Ashfaq Shuaib, Frank Silver, Eric E Smith, \\ Grant Stotts, Michael Suddes, Richard H. Swartz, Philip Teal, Tim Watson, Michael D. Hill
}

doi:10.1017/cjn.2014.41

Can J Neurol Sci. 2014; 41: 694-696

The importance of treating ischemic stroke patients quickly has long been recognized, and the mantra "Time is brain", is now ubiquitous. ${ }^{1,2}$ Unfortunately, the thinking "We still have time in the treatment window..." is occurring too often during the acute stroke code. The treatment window from time of onset is 4.5 hours $^{3}$ in most guidelines, ${ }^{4,5}$ yet there is declining benefit as time elapses. A 1997 National Institute of Neurological Disorders and Stroke (NINDS) Symposium and the subsequent Brain Attack Coalition $^{6}$ set the standard of 60 minute door-to-needle time. ${ }^{7}$ This door-to-needle time was arbitrary but designed to provide a useful metric. It has now been incorporated into both national guidelines and accreditation standards ${ }^{8-10}$ but has been treated more like a guide or a range rather than a hard target. Parkinson's law - "The job expands to fit the time available" - is as true in stroke care as it is in economics. ${ }^{11} \mathrm{We}$ argue that to change this mentality we must revise our target downward to a 30-minute median door-to-needle time.

Today we know that: (1) "Time is brain" in the most urgent way; (2) with attention to systems change, median door-to-needle times of 30 minutes can be achieved; (3) we fail to meet a 60-minute door-to-needle time for most patients in most centres. The sooner blood flow is restored in ischemic stroke, the better the outcomes. ${ }^{12,13}$ For each 15 minute reduction in delay, there is an estimated $4 \%$ improvement in good clinical outcomes. ${ }^{13}$ The number of neurons that are lost in a middle cerebral artery stroke has been quantified to be 1.9 million per minute or 114 million neurons every hour, which translates to 3.6 years in accelerated aging. ${ }^{2}$ While this is based upon an average rate of infarct progression and individual patients may vary substantially, the damage is staggering. Acute ischemic stroke is a medical emergency that is as, or more, time sensitive than myocardial infarction ${ }^{14}$ and trauma. ${ }^{15} \mathrm{We}$ need to treat it as such. Using conservative calculations of 800000 strokes per year in North America, where 40000 are treated with intravenous tissue plasminogen activator (tPA), and 16000 (40\%) of these have a good outcome, an additional 3000 patients will have good outcomes based on a decrease of 30 minutes in time to treatment. ${ }^{16}$ In addition, this calculation does not allow for a projected increase in the number of patients treated. The optimal door-to-needle time is the fastest possible time that preserves safety and appropriateness.

When hospitals and stroke teams use a systematic quality improvement approach to stroke thrombolysis, significantly lower door-to-needle times can be realized. In Helsinki, an initial median door-to-needle time of 1 hour 45 minutes in 1998, was reduced to 50 minutes by $2004,{ }^{17}$ and then further reduced to 20 minutes in 2011 (excluding basilar artery occlusion). ${ }^{18}$ This model was replicated at the Royal Melbourne Hospital, where median door-to-needle times were reduced from 61 minutes to 25 minutes. ${ }^{19}$ Improvements in the United States (US) have been reported by the Target-Stroke initiative. ${ }^{20,21}$ Importantly, these improvements in door-to-needle treatment time have not resulted in an increase in complications. A key concept in fast treatment is the paradigm of parallel (rather than serial) diagnostic evaluation, assessment and treatment. The Emergency literature discusses the concept of "swarming" for acute trauma and acute myocardial infarction. The same must apply to acute stroke.

From the Department of Clinical Neurosciences (NK, MDH, AMD, EES, TW); Department of Emergency Medicine (EL), Faculty of Medicine, University of Calgary; Alberta Health

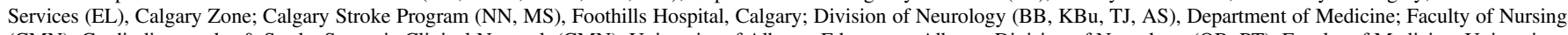

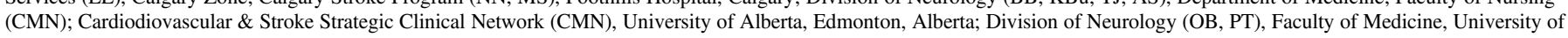

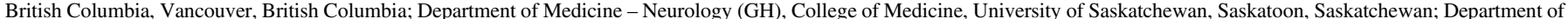

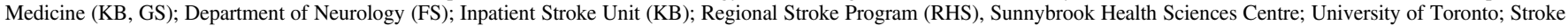

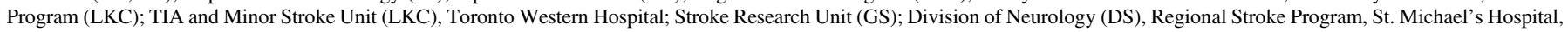

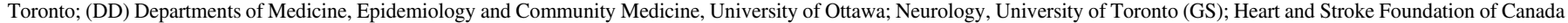
(PL), Ottawa; (AJ) Department of Medicine, Queen's University; Kingston Department of Clinical Neurosciences (JM), Western University, London; Division of Neurology (WO),

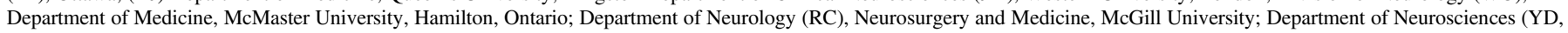

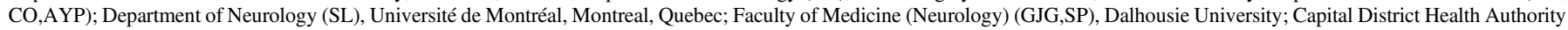
(SP), Halifax, Nova Scotia, Canada.

Received May 5, 2014. Final Revisions Submitted June 12, 2014.

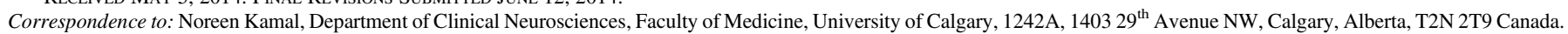
E-mail: nrkamal@ucalgary.ca 
Table: 30 changes to get to 30 minutes

A list of process changes, used at centres across Canada, that may expedite the treatment of acute ischemic stroke with intravenous alteplase

30 Changes to get to 30 minutes: Description of Change

1. EMS involvement in decision making. Ante-cubital IV line placement $\times 2$

2. Pre-hospital notification.

3. Information gathering on electronic systems (electronic medical record, PACS) while the patient is still in the pre-hospital stage

4. Activate ED physicians and nurses at the time of pre-notification.

5. Parallel involvement with stroke team for rapid stabilization and transfer to CT. Fan-out paging of the team: STAT Stroke or Code Stroke page.

6. Fast (even pre-hospital) patient registration into electronic systems

7. Stroke Team includes the ED staff and both are alerted at pre-notification

8. Use of standard work assignments in the stroke ED swarm. Swarm operates like a pit-crew with each task done in parallel.

9. Establish consistent flow - one or two rooms in ED always used as code stroke rooms so team always knows where to go and room set up to facilitate treatment

10. Standardized code stroke note-taking or documentation forms to facilitate information gathering "on the fly"

11. Do not place foley catheter in ED; this can be done after tPA is delivered

12. Large digital timer adhered to the stretcher from the moment of arrival to show the team the time going by

13. Laboratory and CT ordered and informed at pre-notification

14. Standard stroke protocol CT imaging programming

15. No requirement to wait for creatinine prior to imaging

16. Advanced imaging reserved for uncertain cases only

17. Patients transferred directly from triage to the CT table from the ambulance stretcher

18. Do not wait for formal radiology report

19. Do not wait for report of blood test unless there is a specific situational reason (eg. known use of warfarin)

20. Use CT table to obtain the patient weight (if available)

21. tPA delivered in CT immediately after imaging

22. Brief huddle after tPA is administered with the stroke team, ED staff and EMS to discuss what went well and how to improve the process

23. Physician and leadership engagement

24. Targeted continuing professional education

25. Informing/updating every member of the team (EMS, ED, MD, RN, students, technicians laboratory) about the value of reducing door-to-needle times.

26. Regular distribution of reports for DTN times

27. Engagement of hospital and departmental administration

28. Recognition and reward for rapid thrombolysis

29. Pin recognition program for team members treating patients in under 20 or 30 minutes

30. Coffee cards for nurses involved in program change for rapid CT and door-to-needle cases

EMS = emergency medical services; IV = intravenous; PACS = picture archive and retrieval system; ED = Emergency department; $\mathrm{CT}=$ computed tomography; tPA = tissue plasminogen activator; STAT = "immediately" (used in medical contexts from latin "statim"); Code = requiring a team of providers to rush to the specific location and begin immediate treatment; $\mathrm{MD}=$ Medical Doctor; RN $=$ Registered Nurse; DTN $=$ Door-to-Needle

In Canada, comprehensive stroke centres have been able to achieve similar reduction in door-to-needle time. In Calgary, as a result of a six-sigma quality improvement process, the stroke team's median door-to-needle time was reduced to 32 minutes (range 11-121 min) from 52 minutes (range 6-193 min). Comparable magnitude improvements have been seen in Montreal (median $54 \mathrm{~min}$ reduced to $30 \mathrm{~min}$ ), Ottawa (median $74 \mathrm{~min}$ reduced to $37 \mathrm{~min}$ ) and at other Canadian stroke centres. However, both the US and Canada have globally failed to meet the 60-minute door-to-needle target. Results from the American Heart Association "Get With the Guidelines" data show that only $26.6 \%$ of patients received tPA within 60 minutes of arriving at a hospital. ${ }^{22}$ In Canada, results from the 2011 national stroke audit on quality of stroke care showed that only $34 \%$ of patients had a door-to-needle time equal to or less than 60 minutes. ${ }^{23}$

To improve we can learn from success. The process changes that were employed by the groups in Helsinki, Melbourne and our groups in Canada are not novel. [Table] These groups have simply recognized the urgency in hyper-acute stroke care of ischemic stroke. They developed processes to ensure this mindset permeates to Emergency Medical Services (EMS), Emergency Department, and stroke team. They have worked in parallel as a team. Based on results from these groups, we ask: How long does it take to administer tPA? Is a median 60-minute door-to-needle time acceptable? Internationally, other professional groups have recognized that, globally, we are not treating stroke fast enough. ${ }^{22,24}$

We are calling for an aggressive update of our targets as a necessary step to better patient care. Today we should set a door-to-needle benchmark at a 30-minute median (60-minute $95^{\text {th }}$ percentile). To achieve a system where $95 \%$ of patients get treated within 60 minutes, where patients are treated with the fastest possible door-to-needle time, we need a median door-to-needle time of 30 minutes. Systems that aim for and achieve this target will have engineered their processes to allow for predictable and unavoidable variance due to patient factors that can necessarily delay treatment. Centres that can achieve these results will see more of their patients walking out of hospital and will have met an essential criterion for being named a top stroke centre.

This target is not easy but it is demanded by the immutable biology of the disease. These efforts will require ongoing efforts to maintain success. Key implications of such a change could include centralization of acute stroke care to specialized centers where this makes sense geographically, increased use of telemedicine, ${ }^{25}$ a strong emphasis on training to avoid treatment of stroke mimics, and more careful and discriminative use of diagnostic imaging. Success will be achieved in different ways at different centres. For our patients, let us commit to reducing delays in stroke thrombolysis and set a new standard for ischemic stroke treatment. 


\section{REFERENCES}

1. Gomez CR. Time is Brain. J Stroke Cerebrovasc Dis. 1993;3(1):1-2.

2. Saver JL. Time is brain-quantified. Stroke. 2006;37(1):263-6.

3. Hacke W, Kaste M, Bluhmki E, Brozman M, et al. Thrombolysis with alteplase 3 to 4.5 hours after acute ischemic stroke. NEJM. 2008;359(13):1317-29.

4. Bluhmki E, Chamorro A, Dávalos A, et al. Stroke treatment with alteplase given $3.0-4.5 \mathrm{~h}$ after onset of acute ischaemic stroke (ECASS III): additional outcomes and subgroup analysis of a randomised controlled trial. Lancet Neurol. 2009;8(12):1095-102.

5. Sandercock P, Wardlaw JM, Lindley RI, et al. The benefits and harms of intravenous thrombolysis with recombinant tissue plasminogen activator within $6 \mathrm{~h}$ of acute ischaemic stroke (the third international stroke trial [IST-3]): a randomised controlled trial. Lancet. 2012;379(9834):2352-63.

6. Alberts MJ, Latchaw RE, Jagoda A, et al. Revised and updated recommendations for the establishment of primary stroke centers: A summary statement from the brain attack coalition. Stroke. 2011;42:2651-65.

7. Marler JR, Winters Jones P, Emr M. The National Institute of Neurological Disorders and Stroke: Proceedings of National Symposium on Rapid Identification and Treatment of Acute Stroke. Bethesda, MD: National Institute of Neurological Disorders and Stroke; 1997.

8. Schwamm LH.. Primary Stroke Center Certification. Honolulu, HI: International Stroke Conference; 2013.

9. American Stroke Association. Target: Stroke Time Lost is Brain Lost. http://www.strokeassociation.org/idc/groups/heart-public/ @wcm/@hcm/@gwtg/documents/downloadable/ucm_308277. pdf.

10. Casaubon LK, Suddes M, on behalf of the Acute Stroke Care Writing Group. Chapter 3: Hyperacute Stroke Care. In Lindsay MP, Gubitz G, Bayley M, Phillips S (Editors) on behalf of the Canadian Stroke Best Practices and Standards Advisory Committee Canadian Best Practice Recommendations for Stroke Care. 2013. Ottawa, Ontario Canada:Canadian Stroke Network and Heart and Stroke Foundation of Canada.

11. Parkinson NC. Parkinson's law. Economist. 1955.

12. Christou I, Alexandrov AV, Burgin WS, et al. Timing of recanalization after tissue plasminogen activator therapy determined by transcranial Doppler correlates with clinical recovery from ischemic stroke. Stroke. 2000;3:1812-6.
13. Saver JL, Fonarow GC, Smith EE, et al. Time to treatment with intravenous tissue plasminogen activator and outcome from acute ischemic stroke. JAMA. 2013;309(23):2480-8.

14. Rawles J.. Magnitude of benefit from earlier thrombolytic treatment in acute myocardial infarction: new evidence from Grampian region early anistreplase trial (GREAT). BMJ. 1996;312(7025):212-5.

15. Lerner EB, Moscati RM. The golden hour: scientific fact or medical "urban legend"? Acad Emerg Med. 2001;8(7):758-60.

16. Lees KR, Bluhmki E, Von Kummer R, et al. Time to treatment with intravenous alteplase and outcome in stroke: an updated pooled analysis of ECASS, ATLANTIS, NINDS, and EPITHET trials. Lancet. 2010;375(9729):1695-703.

17. Lindsberg PJ, Häppölä O, Kallela M, Valanne L, Kuisma M, Kaste M. Door to thrombolysis: ER reorganization and reduced delays to acute stroke treatment. Neurology. 2006;67(2):334-6.

18. Meretoja A, Strbian D, Mustanoja S, Tatlisumak T, Lindsberg PJ, Kaste M. Reducing in-hospital delay to 20 minutes in stroke thrombolysis. Neurology. 2012;79(4):306-13.

19. Meretoja A, Weir L, Ugalde M, et al. Helsinki model cut stroke thrombolysis delays to 25 minutes in Melbourne in only 4 months. Neurology. 2013;81(12):1071-6.

20. Fonarow GC, Smith EE, Saver JL, et al. Improving door-to-needle times in acute ischemic stroke: The design and rationale for the american heart association/american stroke association's target: Stroke initiative. Stroke. 2011;42:2983-9.

21. Fonarow GC, Zhao X, Smith EE, et al. Door-to-needle times for tissue plasminogen activator administration and clinical outcomes in acute ischemic stroke before and after initiation of a quality improvement initiative. JAMA. 2014;311(16):1632-40.

22. Fonarow GC, Smith EE, Saver JL, et al. Improving door-to-needle times in acute ischemic stroke the design and rationale for the American Heart Association/American Stroke Association's target: stroke initiative. Stroke. 2011;42(10):2983-9.

23. Canadian Stroke Network. The Quality of Stroke Care in Canada. 2011. http://www.canadianstrokenetwork.ca/wp-content/uploads/ 2011/06/QoSC-EN1.pdf.

24. Safe Implementation of Treatments in Stroke. SITS WATCH. 2014. https://sitsinternational.org/sits-projects/sits-watch.

25. Schwamm LH, Holloway RG, Amarenco P, et al. A review of the evidence for the use of telemedicine within stroke systems of care. A scientific statement from the American Heart Association/ American Stroke Association. Stroke. 2009;40:2616-34. 